\title{
Piloting E-Learning in a Radiation Oncology Department
}

\section{ABSTRACT}

Radiation therapy is an ever-evolving profession that requires radiation therapists (RTs) to continually update knowledge and skills in order to provide quality patient care. In 2006, the Radiation Therapy Educators (RTE) at the Calvary Mater Newcastle Hospital piloted the delivery of in-service education using an e-learning format.

In order to investigate the effectiveness of e -learning compared to the traditional face-to-face method of education, the RTEs chose to compare two cohorts of Professional Development Year (PDY) as the sample. The department orientation and treatment planning introduction, were the programs used. The first PDY cohort received these programs using the traditional face-to face method and the following years' cohort were presented the material using an e-learning platform.

Although the project was limited by a small sample size, the results indicated that e-learning delivered the same content in a more efficient manner than face-to-face whilst ensuring the same learning outcomes were met.

\section{INTRODUCTION}

\section{Background}

In our cancer care centre (New South Wales/Australia) clinical educators have the responsibility of not only addressing the clinical needs of undergraduate Medical Radiation Science (MRS) students, but to meet the professional development needs of new MRS graduates undertaking the mandatory PDY and qualified RTs.

The clinical environment is a complex workplace that demands all levels of RTs to provide quality care to ever increasing patient numbers, whilst adapting to the constantly evolving technologies that radiation therapy relies on. In many professions knowledge is constantly changing with the advent of new technologies. The health professions are no exception. Hughes indicates that the life span of nursing knowledge is two to five years and states, "Basic professional education is no longer sufficient for a life time of practice" ${ }^{1}$. Radiation therapy education often looks to medicine, nursing and other allied health professions for application of educational theories and concepts. Therefore, the knowledge that a RT has, can be assumed to have a similar life-span. In response to seemly never ending change in technology and required knowledge, continuing professional development (CPD) has become a necessary requirement for health care professionals in order to maintain clinical competency and ensure there is a strong adherence to evidence-based knowledge and maintaining best-practice standards. CPD is often seen as an individual's responsibility; however health care organizations see the benefits in facilitating it in the workplace. ${ }^{2}$

An ideal scenario of workplace learning ensures both the employer and employee benefit. The employer benefits by ensuring employees deliver a high quality of service and productivity, and the employees benefit by receiving up-to-date information, providing the opportunity for skill improvement and job satisfaction ${ }^{3}$. According to Benson ${ }^{4}$, the availability of learning material in 
the workplace allows the learner to immediately apply the knowledge, enhancing the transfer of knowledge. Merriam and Caffarella describe the main advantage in providing education in the workplace to be that, "Organizations learn only through individuals who learn" 5 . In order for an organisation to provide quality education, workplace educators must have knowledge and skills in adult education. Educators must have knowledge in andragogical concepts such as surface and deep approaches to learning, knowledge construction and cognitive and metacognitive strategies that facilitate adult learning.

The literature describes that, "Learners retain $10 \%$ of what they read, $30 \%$ of what they see, $50 \%$ of what they see and hear and $90 \%$ of what they act on"4. Therefore, the opportunity to interact or actively participate in their education will result in improved learning. In the past, all education delivered within the department has relied on face-to-face didactic methods. However with an increase in student and staff numbers, the RTEs had to source alternative methods of providing efficient, high standard of education and training on a larger scale, with existing educator numbers.

\section{E-learning}

E-learning, computer-assisted/aided instruction, distance learning, blended learning and mobile learning are all terms used to describe the use of interactive web-based programs to deliver educational content. For the purpose of this discussion, the terms e-learning and blended learning will be used. Although literature suggests that a consistent definition regarding elearning is lacking, it has been suggested that the creation of frameworks or taxonomies of elearning strategies would be more useful ${ }^{6}$. This inconsistency is possibly due to the fact that elearning has a relatively short history "and it can be characterized by rapid changes in technology"7. There appears to be some consistency in that there are generally three components of an e-learning program; there is the educational content, the user interface and the learning management system (LMS) ${ }^{8}$.

In keeping with adult learning principles, e-learning is a learner-centered approach; allowing the learner to progress at one's own pace. It provides opportunity for self-directed education and if designed with an interactive component, it has the propensity to improve learning ${ }^{4}$. E-learning can be used in a blended learning approach where there "is the use of two or more distinct methods of training"'.

The benefits of e-learning include "cost-effectiveness, time efficiency, accessibility, use of different instructional strategies and providing consistent information"4; impacting on organisations, staff and ultimately patient outcomes by providing efficient, quality and up to date training for all staff within an organisation. Some of the limiting factors to the development of an e-learning program can include; educator skill level and resources to develop an e-learning package, updating and maintaining the material and initial set up costs.

\section{Application to the clinical setting}

There is a vast amount of published literature available exploring e-learning, however research exploring its use in the clinical setting is limited. Blended learning with e-learning as a component is an attractive option in the clinical setting. In an article by Fernandez ${ }^{10}$, an increase in demand on health facilities, specifically patient numbers and staff shortages, result in managements reluctance to allocate time for training in the workplace. An e-learning program 
can aid in the delivery of theoretical knowledge. When e-learning is combined with face to face delivery, learning can be optimised because individual learning styles can be accommodated and skill development and social interaction provided ${ }^{11}$.

E-learning has great potential to assist all stakeholders in the clinical setting; "E-learning enables health care employees to confidently access, interpret and apply organizational knowledge, patient care procedures, professional work force competencies, best practices knowledge, and other skills information - in a manner that improves patient satisfaction, achieves positive

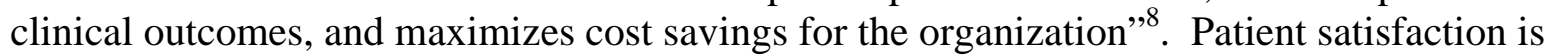
improved as a direct result of CPD ${ }^{13}$. When e-learning is used as a method to provide CPD more time can be spent on service delivery. There are numerous benefits of e-learning in the clinical setting, these include; stream lining content, focusing on what is important and increasing speed of delivery and lowering costs by ensuring the time spent away from daily duties is minimized ${ }^{8}$. Autonomy and flexibility are other advantages over instructor-based education $^{12}$, as is allowing constant availability of material to multiple users at the same time ${ }^{8}$.

The major caution in using e-learning pertains to the mix of staff with varied learning styles and learning preferences, e-learning does not always suit all of the learning styles within a workplace. Hence it is important to embed e-learning into a blended learning package in some environments.

Educators benefit as e-learning enables them to concentrate on the important task of ensuring the transfer of learning is occurring rather than focusing on educating, allowing them to focus on the learners' needs and developing supplementary information ${ }^{11}$. Incorporating e-learning into orientation programs frees up educators and preceptors "to spend more time integrating orientees into their new environment" 12 . According to Benson ${ }^{4}$, nursing educators have found e-learning as effective, if not more than conventional lectures, and when the two are combined this results in improved cognition. E-learning can also aid in resource management allowing educators to track and record educational activities. ${ }^{8}$

Radiation therapy, like nursing, is practiced in the clinical setting. E-learning could provide thorough, consistent training to all staff in a flexible, available delivery system whilst ensuring optimum delivery of patient care. Investigation into e-learning software packages resulted in Knowledge Presenter ${ }^{\circledR}$ being chosen. This system provided a complete package of easy to use authoring software and a LMS which would facilitate delivery, accurate tracking and recording of workplace education and training.

This project aims to compare the resources employed when delivering a previously established PDY department orientation and treatment planning introduction program; utilizing traditional teaching methods, to that when delivered using e-learning in a blended learning environment.

This project includes two cohorts of PDY RTs over two consecutive years. The project compares the resources used once an e-learning program was implemented for the department orientation and training, with that of the previous year when the department orientation and fundamental training was delivered via a conventional didactic and tutorial based approach. The 
resource utilization was measured using face to face hours spent with the PDY therapist and assistance phone calls to the RTEs from the PDY therapist during the training period.

By establishing whether there is a significant difference in the resources used, specifically the time RTEs invested in the training between the two cohorts, it may lead to changes in future and existing education programs within the department in an attempt to maximize efficiency and efficacy in continuing education and professional development in a clinical working environment.

It is important to note that this project does not evaluate the time taken to author the training material for either arm of the project. It has been assumed that regardless of the method there are necessary preparation and authoring requirements.

Each of the PDY RTs were evaluated as part of a year long assessment process to ensure at various stages of their internship that they were meeting their objectives and achieving their learning outcomes. The assessments were completed by a senior RT every six weeks, who was independent to the project. It was assumed that if the PDY therapist was able to proceed following each summative assessment the learning outcomes were being met regardless of the delivery method. Hence, it was not necessary for this project to reassess whether a specific level of competence was achieved by each participant

\section{METHOD}

\section{Training Delivery}

Traditional method-The department's orientation and planning fundamental modules were delivered using a combination of didactic presentations, reading modules and exercises.

E-learning-This arm involved transforming the training modules mentioned above into material that could be delivered in an e-learning format, utilising authoring software called Knowledge Presenter ${ }^{\circledR}$. This arm of the project delivered the same content and employed the same exercises as the traditional arm, simply using an electronic platform. Similar to when the traditional package was developed, the e-learning package did utilize educator resources; however this was no more than that required for the traditional package and was only required in the initial development stage. The skills necessary for the development of the e-learning package were familiar to the RTEs and not specific to e-learning or computer coding.

\section{Sample}

The sample included two cohorts of students both graduating from MRS Radiation Therapy undergraduate degree programs from different universities across Australia, who were successful in gaining a PDY position within the department. The cohort that received the training via the conventional method contained 4 PDYs, whilst the cohort receiving the training via e-learning contained 5 PDYs.

To be eligible to participate in the PDY program the participants had completed a Bachelor of Applied Science in Medical Radiation Science, Radiation Therapy. This is a three year undergraduate program consisting of both theory and clinical component. Effectively the PDY is 
an intern year where the therapist does not work unsupervised in any form. On completion of the PDY program the therapist is eligible for full accreditation with the Australian Institute of Radiography (AIR).

\section{Data collection and analysis}

The data collected was the same for both cohorts. The number of phone calls to the RTEs for assistance whilst completing the training program was collected for each participant, as were the number of face to face hours the RTEs spent with each participant whilst completing the program. There was no need for assessment of the level of competence met at the end of the program, as all PDYs must meet standards set by the AIR Professional Accreditation and Education Committee (PAEC), after completing their PDY regardless of delivery styles employed at each centre.

To ensure that face to face hours were not substituted by extended phone calls, help desk calls were limited to 5 minutes. For phone calls exceeding this time limit a face to face session would be initiated. The help desk calls were initially recorded on hard copy log sheets and then entered into a MS Excel ${ }^{\circledR}$ spreadsheet for analysis. This data was retrieved from an established quality assurance system that records daily statistics for the clinical educators within the department. These statistics detail the content and duration of the clinical educators activities for each day. All data entry was independently checked to ensure accuracy prior to analysis.

The data was examined descriptively calculating frequencies to compare the resource usage for both delivery methods. Although the sample size was very small, the data was analysed for both statistical and clinical significance. The data was skewed so the median and range were reported and groups were compared using non-parametric analysis (Mann-Whitney-U test).

\section{RESULTS}

The median number of hours of face to face in the e-learning group was 4.5 (range 3.00-6.50) hours compared with a median of 28.13 (range 9.70-42.00) hours in the conventional group (figure 1).

The median number of phone calls in the e-learning group was zero (range 0-6) hours compared with a median of eight (range 2-18) hours in the conventional group (figure 2). There was a significant difference between the groups in the number of hours of face to face education $(Z=-$ 2.46, $\mathrm{p}=0.14$ ) but not in the number of phone calls $(\mathrm{Z}=-1.50, \mathrm{p}=0.13)$.

\section{DISCUSSION}

The pilot project experienced an unavoidable small sample size, as it was limited by the number of PDY RT positions available in the department. In a review of the published literature available on e-learning within the clinical context, there is no precedent that supports nor refutes this sample size. Regardless this small sample has produced a statistically significant result in the difference between the face to face hours spent with the PDY RTs in the e-learning arm versus the conventional arm. This significant reduction in consumption of the RTEs time as a 
result of the e-learning delivery method shows the ability of e-learning programs to free up educators time, allowing them to focus on other departmental tasks, as well as on areas that staff and students identify as problematic or difficult areas. Hence, RTEs can be used more effectively to ensure that the PDY RTs is not only receiving all the information they need but that educators have the time to assist when clarification or intensive training is necessary; rather than utilizing valuable time on delivery of standard and routine information. Although there was no statistical significance in the difference between the number of phone calls for assistance in the e-learning to conventional delivery cohorts, there was a great clinical significance identified by the RTEs. There was a great reduction in the phone calls received from the PDY RT in the elearning cohort compared to the conventional cohort whilst undertaking the training, which impacts greatly on the resources in a clinical department. Within the conventional cohort, there is a large difference in help desk phone calls among the participants. Participants A and D had a large number of help desk phone calls and a smaller number of face to face hours. Whilst participants $B$ and $C$ are the reverse of this. As previously discussed, once a phone call exceeded five minutes, a face to face session was then initiated and it was logged accordingly. With participant $B$ and $C$ requiring more face to face work, there were less logged calls than participant $\mathrm{A}$ and $\mathrm{D}$. This difference may be attributed to by the learning style or previous experience in the topic area of the individual participants.

Since the completion of this evaluation, the use of e-learning has expanded to include all areas of low level planning and treatment planning software training with a recent development of an Eclipse treatment planning training package; as well as an electronic reflective journal club. The future of this initiative within the department is limitless. There has been a large amount of interdisciplinary interest as well as the inclusion of a Brachytherapy program. It has also been identified for use in medical and physics registrar training, and as a platform for continuing anywhere, anytime access to all staff for training, development and currency of skills and knowledge.

The e-learning program within the department is still in its' infancy and increasing the range of users to include all staff levels, ages and learning styles may prove challenging. With the right balance of blended learning techniques involving group trouble shooting sessions, availability of print media and a comprehensive e-learning program this method of content delivery provides an exciting prospect for training and education with the department.

\section{REFERENCES}

1. Hughes P. Evaluating the impact of continual professional education. Nurse Education Today. 1990.10: p.428-436.

2. Ryan J. Continuous professional development along the continuum of lifelong learning. Nurse Education Today. 23(7),2003.23(7): p.498-508.

3. Spencer B. Research and the pedagogics of work and learning. Journal of Workplace Learning. 2002. 14(7): p.298-305.

4. Benson E. Staff development. Online learning: a means to enhance professional development. Critical Care Nurse. 2004.24(1):p.60-3.

5. Merriam S, Caffarella R. Learning in Adulthood. 1999, San Fancisco: Jossey-Bass. 
6. Servage L. Strategizing for workplace e-learning: some critical considerations. Journal of Workplace Learning. 2005.17(5/6): p.304.

7. Tynjälä P, Häkkinen P. E-learning at work: theoretical underpinnings and pedagogical challenges. Journal of Workplace Learning. 2005.17(5/6): p.318.

8. Nelson E. E-learning. A practical solution for training and tracking in patient-care settings. Nursing Administration Quarterly. 2003. 27(1): p. 29-32, 2003 Jan-Mar.

9. EPIC, Blended Learning. Epic Group Plc White Paper, 2003: p. 1-44.

10. Fernandez J. E-learning replaces classroom. Wisconsin healthcare network uses LMS to educate staff and to track federally mandated training. Health Management Technology. 2003. 24(4): p. 50-2, 2003 Apr.

11. Berke WJ, Wiseman TL. The e-learning answer. Nursing Management. 2003. Supplement: p. 26-9, 2003 Oct.

12. Huryk LA. Enhance orientation through e-learning. Nursing Management. 2005. 30(1): p. 48-9, 2005 Jan.

13. Field N, Mandatory Continuing Professional Education: Do we need it? 2004. 51(1): p.59, 2004 Apr.

Figure 1. Face to face hours spent with RTE for each PDY therapist in conventional and e learning cohorts.

\section{Face to Face Hours with RTE}

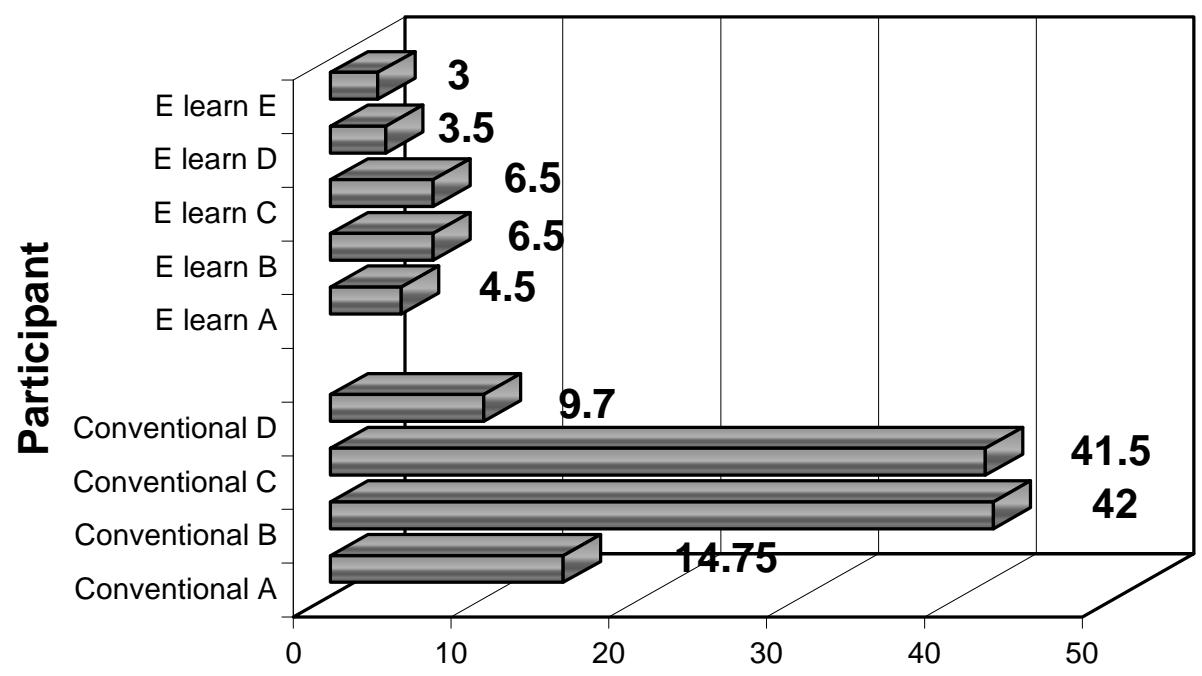

Hours 
Figure 2. Assistance phone calls to a RTE for each PDY therapist in conventional and e learning cohorts

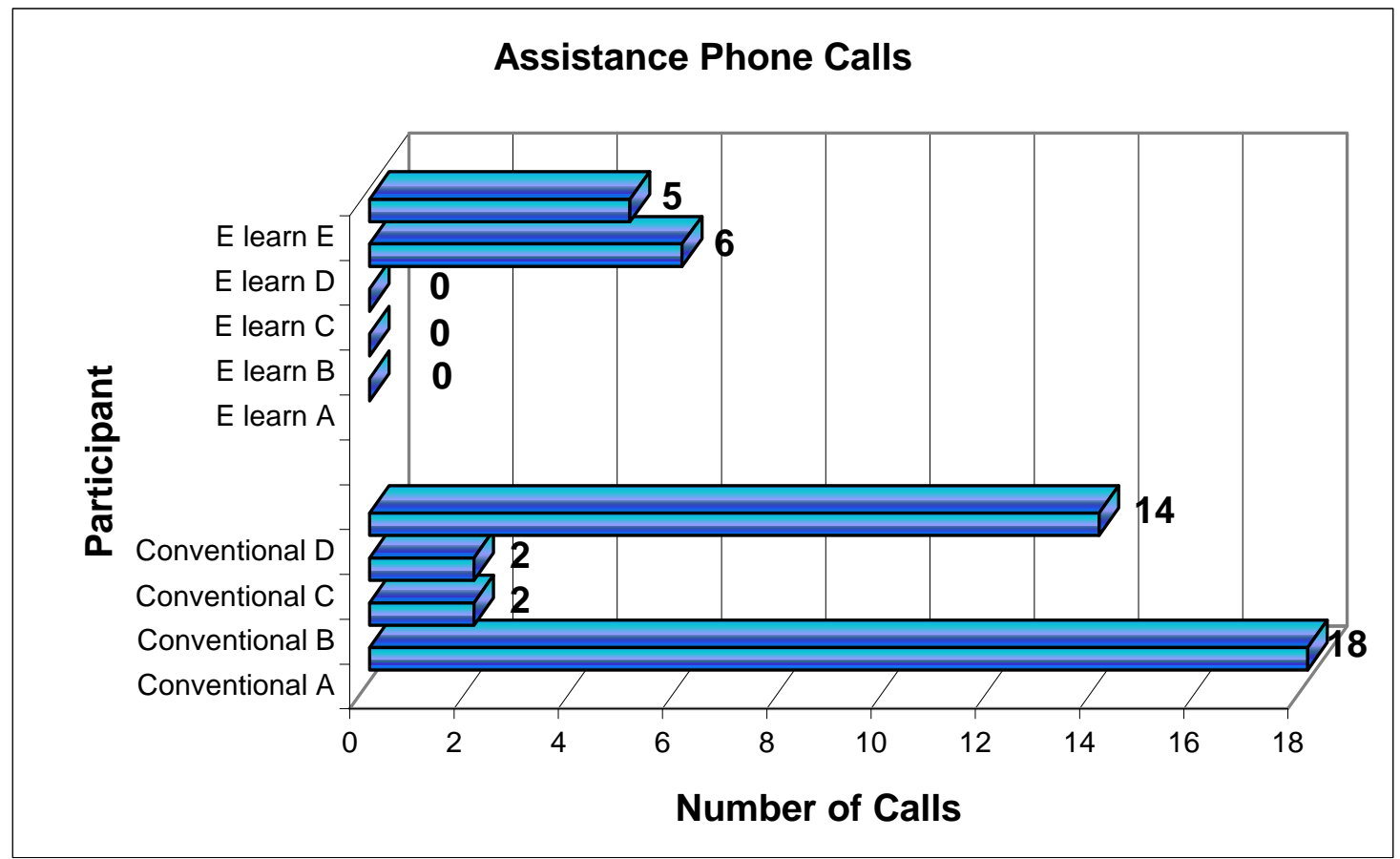

